\title{
1 Mapping malaria risk in low transmission settings: challenges and opportunities
}

3 Hugh JW Sturrock, Adam F Bennett, Alemayehu Midekisa, Roly D Gosling, Peter W Gething, Bryan

4 Greenhouse

5 Correspondence to: Hugh.Sturrock@ucsf.edu (Sturrock, H.J.W.)

7 Abstract

8 As malaria transmission declines, it becomes increasingly focal and prone to outbreaks. Understanding

9 and predicting patterns of transmission risk becomes an important component of an effective

10 elimination campaign, allowing limited resources for control and elimination to be targeted cost-

11 effectively. Malaria risk mapping in low transmission settings is associated with some unique challenges.

12 This article reviews the main challenges and opportunities related to risk mapping in low transmission

13 areas including recent advancements in risk mapping low transmission malaria, relevant metrics and

14 statistical approaches and risk mapping in post elimination settings.

\section{Malaria risk mapping}

17 The sophistication of approaches to mapping malaria risk has grown dramatically over the past 15 years

18 [1-5]. This has enabled spatio-temporal patterns of risk to be quantified with progressively more

19 accuracy and at finer levels of detail, allowing for improved national and global estimates of burden and

20 of populations at risk of malaria, facilitating resource allocation. Understanding spatio-temporal

21 patterns of risk is particularly important in low transmission settings, where malaria transmission

22 becomes increasingly rare, often clusters into hotspots [6-9], and results in outbreaks. In order to

23 remain cost-effective, programs in these settings need to transition from universal coverage of

24 interventions to a more targeted approach. This shift in approach is especially relevant and important

25 for malaria elimination programs which often face dwindling resources as the malaria burden declines. 
26 Metrics and methods to map malaria risk in moderate and high transmission settings are well

27 established. Far less attention has been given to low transmission settings, where traditional metrics

28 used for risk mapping become less useful. An estimated 25\% (811 million) of the total global population

29 at risk of malaria in 2014 live in one of the 35 malaria eliminating countries [10, 11], with many more

30 living in low transmission areas of countries in the control phase. Risk mapping in low transmission areas

31 can support improved targeting of remaining clusters of malaria and help programs reduce malaria

32 burden and eliminate the disease. However, the issue of how to produce risk maps in low endemic and

33 elimination settings requires attention. It is also not clear how malaria risk mapping should be

34 conducted in areas once local transmission has been interrupted. Yet, risk maps are needed in settings

35 where malaria has been eliminated but remain receptive to malaria (areas that have the potential for

36 malaria transmission because the vector is present) or are vulnerable to transmission (areas that have a

37 high rate of importation of malaria parasites in human migrants). Here we review available metrics for

38 evaluating malaria risk in low transmission and post elimination settings, and describe the assumptions,

39 limitations and optimal modeling approaches when using each to estimate and predict risk.

\section{$41 \quad$ Available transmission metrics for low transmission risk mapping}

42 Entomological Inoculation Rate

43 There are a number of indicators which act as proxies for transmission (Table 1). M any of these measure

44 different aspects of transmission and are related to each other, albeit in complex and nonlinear ways

45 [12]. The entomological inoculation rate (EIR) is considered by some to be the gold standard for

46 estimating transmission. In practice, measurements of EIR are labor intensive and difficult to

47 standardize, requiring capture of sufficient numbers of mosquitos and subsequent examination for

48 sporozoites. EIR is particularly challenging, if not impossible, to estimate accurately in low transmission

49 settings, where the density of mosquitoes can be extremely low [13, 14]. Finding a malaria vector, yet

50 alone an infected vector, is near impossible in many low transmission settings. Other mosquito based

51 indicators, such as vector presence, density, and human biting rate are not directly correlated to

52 transmission although they are useful for program decision making [12].

53 Parasite rate 
54 Analyses of spatio-temporal patterns of malaria transmission have commonly focused on the use of 55 parasite rate (PR, infection prevalence) data $[1,5,15]$. Parasite rate is relatively easily captured via 56 cross-sectional surveys, using microscopy, rapid diagnostic tests (RDT), or molecular diagnostics.

57 Furthermore, studies have shown a predictable relationship between PR and EIR [16]. As transmission declines, however, the sample sizes required to generate risk maps with acceptable uncertainty become 59 unfeasibly large, even if surveys are targeted to areas of transmission or when more sensitive molecular 60 diagnostics are used to detect infections $[17,18]$. For example, the Swaziland malaria indicator survey 61 (M IS) conducted in 2010 collected blood samples from 4330 individuals, with only one Plasmodium 62 falciparum infection found by polymerase chain reaction (PCR) [19]. The point at which PR is no longer a 63 suitable indicator for measuring and mapping transmission is dependent on a number of factors

64 including the financial and operational implications of sample sizes required; historically when prevalence is $<3 \%$ alternative metrics, such as clinical incidence, have been advised [20].

66 Serology

67 Serological methods - the detection of human antibodies against malarial parasites - have the potential 68 to address some of the limitations of PR in low transmission settings. Antibody responses to Plasmodium 69 antigens provide a record of exposure to parasites, providing information about past as well as present 70 transmission. As such, evaluation of antibody responses in a community provides a more sensitive 71 measure of exposure that can be mathematically converted into estimates of transmission and may 72 allow for more precise estimates and/or smaller sample sizes than those obtained from PR in low and 73 very low transmission settings. Studies have shown that antibody seroconversion rates, derived from 74 age-stratified seroprevalence data, correlate well with EIR across a wide range of transmission and are 75 able to detect changes in transmission over time [21, 22]. Newer analytical methods have recently been 76 proposed to take full advantage of information reflecting antibody titer often available in the laboratory 77 data, instead of reducing responses to binary values by choosing a cutoff, the choice of which is not 78 always straightforward [23, 24]. These methods may offer increases in precision, but require further 79 validation and are dependent on being able to consistently transform raw data (e.g. optical density or 80 mean fluorescence intensity) into relative quantification of antibody titer.

81 A limitation of most established serological methods is that the traditional antibodies examined (e.g. 82 apical membrane antigen 1 (AM A 1) and merozoite surface protein 1 (M SP1)) do not distinguish very 83 recent exposure (e.g. within the last year) from more distant exposure. Changes in exposure over time 84 may leave their mark on the shape of the relationship between seroprevalence and age, but these are 
most easily detectable if changes occur over a relatively discrete time period and exposure is well distributed across age groups. Thus, while established serological methods are useful for inferring historic and medium-term risk of exposure to parasites, their utility for mapping current risk is dependent on the spatio-temporal stability of malaria transmission, i.e. the persistence of transmission at a given location over time. This is because evidence of historic exposure is not useful in detecting transient hotspots, by definition, and also because individuals' higher cumulative exposure within a stable vs. transient hotspot is more likely to result in a detectable antibody signal. Evidence from moderate transmission settings suggests that at least some transmission 'hotspots' are relatively stable over time $[8,25,26]$. Similar evidence from low transmission settings is lacking, although data from Swaziland, a country on the verge of malaria elimination, suggests that while local cases appear to be confined to the wetter, low lying areas of the country, there is considerable geographic variation between years. While serological 'hotspots' identified during the 2010 M IS have accurately predicted some areas of previously unidentified risk, many cases also appear outside of these areas (Figure 1).

One approach to extend the utility of serological measures for predicting transmission is to model them with environmental and climatological variables, as done recently in Ethiopia [27]. That said, as established serological markers are unable to cleanly distinguish between historic and recent exposure, it remains unclear at what temporal resolution the data represent. New serological markers and analytical methods that are able to more precisely quantify exposure over different timescales, including measurement of very recent exposure, offer an exciting potential to better understand spatio-temporal patterns of risk [28, 29]. Different antigenic targets may also provide qualitatively different information. For example, antibody responses against a parasite protein exclusively produced during the preerythrocytic stage of the life cycle will be more likely to reflect the timing of mosquito to human transmission, while responses against blood stage parasites will also be influenced by the duration of chronic infections.

The use of serology for mapping current transmission risk in low transmission settings shows promise, but requires further evaluation and development of sensitive and specific markers of very recent exposure. That said, serology has the unique attribute of being able to provide retrospective information on transmission patterns. Once a sero-marker for recent exposure in low transmission settings can be rigorously validated, serology can easily and cheaply be incorporated into monitoring and evaluation efforts and can be used to evaluate the impact of interventions [30, 31]. 
116 An alternative to the aforementioned approaches is to use clinical surveillance data, such as annual

117 parasite index (API) which refers to the numbers of clinical cases of malaria reported at health facilities

118 per person per year. This metric has the advantage that it is routinely collected as part of passive

119 surveillance systems, in contrast to EIR, PR, and serology which require some level of dedicated active

120 surveillance activities. In moderate to high transmission settings the relationship between API and

121 transmission intensity is confounded by the relationship between exposure and acquired immunity. In

122 low transmission settings, unless there have been very rapid declines in transmission, the majority of the

123 population are likely to have little clinical immunity against symptomatic disease. In these settings API is

124 likely to be more closely associated with transmission intensity [32]. Additionally, the continuous

125 collection of clinical surveillance data means that maps can be updated in real time, providing up to date

126 information on where transmission is currently occurring. This is particularly useful in environments

127 where transmission is not spatially stable over time, such as those prone to epidemic transmission.

128 There are several factors that affect the accuracy with which API reflects transmission risk. First, routine 129 case data often don't differentiate between clinically diagnosed cases and those confirmed by diagnostic 130 tests. Fortunately, since the launch of WHO's Test Treat Track (T3) campaign

131 (http://www.who.int/malaria/publications/atoz/test_treat_track_brochure.pdf?ua=1), the proportion

132 of cases that are correctly confirmed via diagnosis with RDT or microscopy is increasing.

133 Second, information on whether identified cases are acquired locally or are imported is often not

134 available. Since risk maps ideally reflect local transmission, failure to make this distinction when

135 generating risk maps could lead to spurious results. This issue is particularly important in very low

136 transmission settings, where the proportion of cases that are imported may be substantial [33, 34].

137 Where cases are classified by origin, e.g. as estimated by recent travel history, it may be possible to use

138 mathematical modeling to estimate receptivity and malariogenic potential [35], although these methods

139 require further validation and a mechanism to incorporate infections undetected by existing surveillance

140 systems. Incorporating information on human movement may provide a mechanism to estimate

141 importation rates and adjust risk maps generated using passive and/or active surveillance data

142 accordingly. Such an approach has been taken at the national scale in Kenya and Namibia [36, 37].

143 However, estimating international human movement is challenging and movement patterns of the

144 population at large may not be representative of those individuals importing malaria infections. The

145 ability to track parasite movement by genotyping may soon offer an additional solution to understand 
146 parasite population structures and origins of infections, but work to establish the optimal sampling

147 strategies, genetic markers, and analytical methods is in its infancy [38].

148 Third, data reporting is often incomplete, leaving gaps in space and time. Recent technological

149 innovations, such as the introduction of mobile technology (Short M essage Service (SM S), Unstructured

150 Supplementary Service Data (USSD) and internet), have helped to improve completeness and timeliness

151 of reporting, but these tools are not yet widely implemented [39-41]. Where data are incomplete at

152 random (spatially and temporally), this can be accounted for during the modeling process [42, 43]. If,

153 however, data are missing in a more systematic way, analyses need to be interpreted with caution or

154 restricted to areas for which the data quality allow.

155 Fourth, since not everyone with malaria will seek treatment, due to individuals being asymptomatic or

156 unwilling or unable to seek treatment when symptomatic, passive surveillance data typically

157 underestimate true burden. As treatment seeking behavior may vary spatially, applying blanket

158 corrections to country level data is likely to be overly simplistic. More detailed spatial patterns of

159 treatment seeking behavior can be estimated from data collected as part of population level surveys

$160[44,45]$. Such data allow a more accurate estimate of the denominator from which clinical incidence is

161 estimated, in turn improving risk estimates. Where estimates of the population denominator are

162 thought to be uncertain, the test positivity rate (proportion of malaria tests that are positive) (TPR) may

163 be a useful alternative metric to use. While open to influence from variations in testing rates between

164 facilities, and trends in non-malarial fevers, TPR has shown to have a good correlation with other

165 transmission metrics $[46,47]$.

166 Fifth, while some countries now collect and report clinical incidence data from community health 167 workers, routine case data are often only available in aggregate form, i.e. at the level of the health 168 facility or district. This is occasionally true for other types of data as well, but is a particular issue for 169 incidence data since these data collection systems have not been purposefully designed to map risk.

170 Consequently, researchers and control programs are often limited to modeling and predicting incidence

171 at this spatial scale $[39,48,49]$. While mapping at coarse scales offers insight into broader scale

172 transmission patterns of malaria, this approach ignores the inherent heterogeneity of transmission

173 within health facility catchments or districts, precluding identification and targeting of hotspots and

174 associated risk factors at smaller scales. Sturrock et al. [45] adapted a Bayesian hierarchical approach

175 originally applied to species distribution modeling [50] to produce fine scale malaria risk predictions

176 from health facility level data in Swaziland [45]. A similar approach using log-Gaussian Cox process 
177 models, which model the underlying fine scale risk as a spatially continuous intensity surface, has been

178 applied to aggregate level data for other diseases and may be appropriate for mapping malaria as well $179[51,52]$.

180 Where household or village locations of individual cases are available generating high resolution risk 181 maps is theoretically more straightforward. Using random forests models [53], Cohen et al. produced

182 high resolution risk maps of malaria in Swaziland using individual case data [54]. Tatem et al. took a

183 similar approach to produce a risk map in Namibia [37]. For these types of models to be used,

184 information on controls is required. As data on the locations on controls (i.e. individuals known to not

185 have malaria) are not typically available, this requires the generation of "pseudo-controls" or 186 background points. The selection of pseudo-controls requires careful thought and is a focus of 187 investigation [55,56]. Pseudo-controls can be generated by sampling points with probability 188 proportional to the population density to reflect the distribution of the underlying population [54]. 189 However, as noted above, spatial variation in treatment seeking behavior has to be accounted for to 190 ensure that controls are representative of the population from which cases arose.

191 Another issue related to the use of models that require pseudo-controls is that prediction values 192 represent a relative rather than absolute measure of risk and are therefore difficult to convert to or 193 compare with traditional epidemiological metrics. Furthermore, this index of suitability is influenced by 194 the number of background points used (Figure 2). This has been well described by others [57] and is a 195 familiar issue in the analysis of traditional case-control data [58]. An alternative approach is to consider 196 the case data as a realization of a point process and use models designed specifically for these types of 197 data [59]. Point process models attempt to estimate the intensity surface from which the cases arise 198 (see [60] for an excellent review) and therefore allow a direct measure of risk without the need for 199 control data. While point process models have been used for the study and prediction of other diseases 200 [61], to our knowledge they have yet to be applied to malaria data.

\section{Risk mapping post-elimination}

203 In addition to mapping current risk based upon active and passive infection data, in areas approaching 204 elimination and those that have recently eliminated, risk mapping is an important tool for understanding 205 the susceptibility of an area to resurgence or reintroduction. The risk of reintroduction of malaria has 206 historically been evaluated using the concept of "malariogenic potential", which is itself the product of 
207 receptivity and vulnerability [62-64]. Receptivity refers to the overall transmission potential of an area

208 based upon the presence and abundance of anopheline vectors and ecological and climatic conditions permitting parasite development [63]. Vulnerability, on the other hand, refers to the propensity of an area to exposure to new infections, via imported infections or infected mosquitoes [34, 65].

211 Receptivity has traditionally been operationalized using the basic reproductive number, $\mathrm{R}_{0}$, to represent

212 underlying transmission potential in the absence of control, and $\mathrm{R}_{c}$, the reproductive number under

213 control. Both $R_{0}$ and $R_{c}$ are difficult to measure precisely, and are usually modeled based upon existing

214 measurements of vectorial capacity, EIR, parasite prevalence, and incidence [64].

215 Mapping $\mathrm{R}_{0}$ or intrinsic transmission potential can be estimated using historical transmission data, as in

216 Noor et al [66], who used historical maxima in parasite prevalence in northern Namibia to predict

217 maximum transmission potential at $5 \times 5 \mathrm{~km}$ resolution. However, the authors were unable to incorporate

218 data predating the introduction of vector control efforts and therefore likely underestimate true

219 transmission potential. That said, this approach allows a proxy for historical transmission potential,

220 especially in areas with historical data predating control. Changes in the intrinsic transmission potential

221 over time due to climate, economic, ecological, and population changes can only be estimated if high

222 resolution data on these factors can be linked in space and time to historical infection data.

223 Receptivity can also be estimated and mapped from measures of presence and density of anopheline 224 mosquitoes, using the concept of vectorial capacity. Vectorial capacity is an expression of the number of 225 potentially infective bites that originate daily from a case of malaria, and can be measured in a given 226 area from the longevity and density of vectors. Romi et al [67] assessed the receptivity of central Italy 227 using collections of larval and adult anophelines, models of seasonal climatic suitability, and calculation 228 of vectorial capacity. Using these data the authors produced predictive weather-based maps of the 229 distribution of adult anophelines. A number of other studies have produced maps of predicted 230 anopheline density based upon models of larval data, human biting rates, and satellite derived remotely 231 sensed data $[68,69]$.

232 One methodological issue that must be considered when modeling and mapping vector data is the 233 sampling approach, given that vector data are traditionally preferentially sampled in locations where 234 vectors are known to exist. Such preferential sampling is particularly common in low transmission 235 settings where anopheline densities are very low and often highly clustered. Entomological sampling in 236 such settings is therefore only cost-effective if targeted in space and time. If not accounted for during 
237 the modeling process, this sampling bias can impact predictions [70, 71]. Using simulations and

238 observational biomonitoring data from Spain, Diggle et al. approached this problem by considering the

239 sampling locations as an inhomogeneous point process dependent on the spatial process of interest

240 [71]. In turn, this allows unbiased estimation of the spatial process itself. Pati et al. applied similar

241 methods to environmental data in eastern USA [72].

242

243 Given receptivity, the vulnerability of an area to reintroduction of malaria is dependent upon the rate of 244 imported infections into an area. Introduction of parasites into receptive areas, i.e. areas with 245 populations of viable mosquito vectors, can cause resurgence events, as seen in Greece, Sri Lanka, 246 Turkmenistan, and Zanzibar [34]. Understanding which areas are most vulnerable (i.e. prone to 247 importation of new parasites) is also vital for post-elimination control efforts. Basic estimates of 248 vulnerability can be established using proximity to transmission areas as well as by recording the 249 locations where imported infections reside. More complex estimates of parasite movement and 250 vulnerability can be inferred from human movement data and transmission models [37, 73]. Survey data 251 that include travel histories can provide an estimate of the proportion of individuals in an area traveling 252 to other areas with transmission, which can then be used to map estimates of vulnerability at finer 253 spatial scale. Cell phone data, where available, can help to generate estimates of population movement 254 between areas including linkages between areas without transmission and those with transmission ([36, 255 37]). However these data currently only allow estimates of movement within national boundaries as 256 many cell phone providers do not cross national borders, and their spatial scale is dependent upon the 257 density of cell phone towers.

\section{Concluding Remarks and Future Perspectives}

263 While the strengths and weaknesses of different potential metrics have been discussed, in practice, the 264 optimal indicator for risk mapping will depend on the quality and reliability of the available data in any 265 given setting. Despite improvements in the quality of routine case data over the last decade, in many 266 settings the data are of insufficient quality to be reliable. M oving forward, where possible, programs 
267 operating in low transmission settings should consider collecting information which can be used to 268 provide location information on cases. For example, in Thailand and Zanzibar, village name is recorded 269 at the facility and linked to a georeferenced database of villages [40]. In elimination provinces in the 270 Solomon Islands and Vanuatu, cases are linked to a georeferenced database of households at the time 271 of diagnosis [74]. In other low transmission settings, such as Swaziland, Zambia and Zimbabwe, cases are 272 geolocated using GPS devices during active case investigation at household level [6, 75]. Collecting 273 location information on controls, i.e. those who test negative, can also be useful and may help to better 274 understand spatial variation in treatment seeking rates as well as map and predict test positivity rates.

275 Collecting information on travel history, which can be used to distinguish local from imported cases, is 276 critical to distinguish patterns of receptivity from vulnerability. Ideally case classification should be done 277 at the time of diagnosis so as to avoid missing data. While there is currently no standardized method to 278 classify cases as local or imported, recent efforts to identify the origin of infections using parasite 279 genotyping may offer a gold standard against which different approaches can be tested [76-78]

280 An additional approach to improve the utility of routinely collected data for risk mapping is to establish a 281 network of sentinel surveillance sites. This would involve selection of a set of facilities at which 282 enhanced data collection is conducted and rigorously monitored for quality, with additional training and 283 support provided where required [79]. While this will result in sparser data spatially, improving the 284 quality and amount of information collected at sentinel sites would allow for more accurate predictions 285 from spatial models. Additionally, sentinel sites provide examples of best practices which can positively 286 influence the wider health system. Sentinel sites could be randomly selected, or could be more carefully 287 selected to provide an optimal spatial configuration for spatial modeling, as done in environmental 288 monitoring [80].

289 In settings where both serology and case data are of good quality, it may be possible to combine these 290 indicators to allow for a more complete picture of transmission. Similar efforts have been applied to 291 schistosomiasis [81] and loaiasis [82], whereby different indicators resulting from different diagnostic 292 approaches were modeled together to produce predictions of infection prevalence.

293 For countries in both elimination and prevention of reintroduction stages, obtaining robust 294 entomological data relating to the distribution and densities of vectors is central to the long term 295 success of campaigns. Given the low densities of mosquitos, targeted surveillance at high risk times and 
296 locations is likely to represent the most cost-effective approach, but requires careful interpretation and 297 analyses to account for the inherent sampling bias.

298 Generating malaria risk maps in low transmission settings is challenging. Infection prevalence, the

299 indicator most widely used to describe spatial patterns of transmission, becomes increasingly

300 impractical due to sample size requirements. Vector based indicators are challenging for similar reasons.

301 While current serological indicators are useful for describing spatial patterns of historic, long term risk,

302 their utility for targeting resources for prevention of transmission are uncertain. That said, identification

303 of antibody markers of very recent transmission offer exciting opportunities for more dynamic and finer

304 temporal scale risk mapping. Undoubtedly, routine passive surveillance data will continue to play an

305 extremely important role in describing and predicting patterns of low transmission risk, yet these data

306 currently suffer from issues of quality and detail. While surveillance data are steadily improving across

307 countries, there is a need to incorporate and consider a spatial dimension. Having mechanisms and

308 protocols to assign spatial information to surveillance data, such as village or household location of

309 individuals, extends the usefulness of surveillance data and opens up the possibility of describing and

310 predicting transmission risk at high spatial resolution. As programs head towards and beyond

311 elimination, and are faced with dwindling resources and rarer and increasingly clustered hotspots of

312 transmission, capacity to map malaria risk and target resources becomes an invaluable resource to

313 ensure program success.

314 
315 Table 1. Suitability of different indicators of malaria transmission for risk mapping in low transmission

316 settings

\begin{tabular}{llll}
\hline Indicator & Advantages & Disadvantages & $\begin{array}{l}\text { Potential } \\
\text { suitability for } \\
\text { low transmission } \\
\text { risk mapping }\end{array}$ \\
\hline Entomological & $\bullet$ & & Low \\
inoculation rate & Considered by some & $\bullet$ & Operationally \\
& to be gold standard & challenging and \\
transmission metric & expensive to obtain & \\
& & Only provides picture at \\
& & one point in time &
\end{tabular}

\begin{tabular}{|c|c|c|c|c|c|}
\hline $\begin{array}{l}\text { Parasite } \\
\text { prevalence }\end{array}$ & & $\begin{array}{l}\text { Well established and } \\
\text { widely used method } \\
\text { with established } \\
\text { statistical methods } \\
\text { and comparisons over } \\
\text { time } \\
\text { Straightforward to } \\
\text { obtain via cross- } \\
\text { sectional surveys }\end{array}$ & $\bullet$ & $\begin{array}{l}\text { Sample size } \\
\text { requirement in low } \\
\text { transmission often } \\
\text { prohibitively large, } \\
\text { especially to obtain } \\
\text { spatial resolution } \\
\text { required for risk } \\
\text { mapping } \\
\text { Only provides picture at } \\
\text { one point in time } \\
\text { Cross-sectional surveys } \\
\text { can be expensive, done } \\
\text { infrequently }\end{array}$ & Low \\
\hline Serology & & $\begin{array}{l}\text { Ability to integrate } \\
\text { information on }\end{array}$ & $\bullet$ & $\begin{array}{l}\text { M ethods to distinguish } \\
\text { very recent from more }\end{array}$ & $\begin{array}{l}\text { Medium (with } \\
\text { currently }\end{array}$ \\
\hline
\end{tabular}




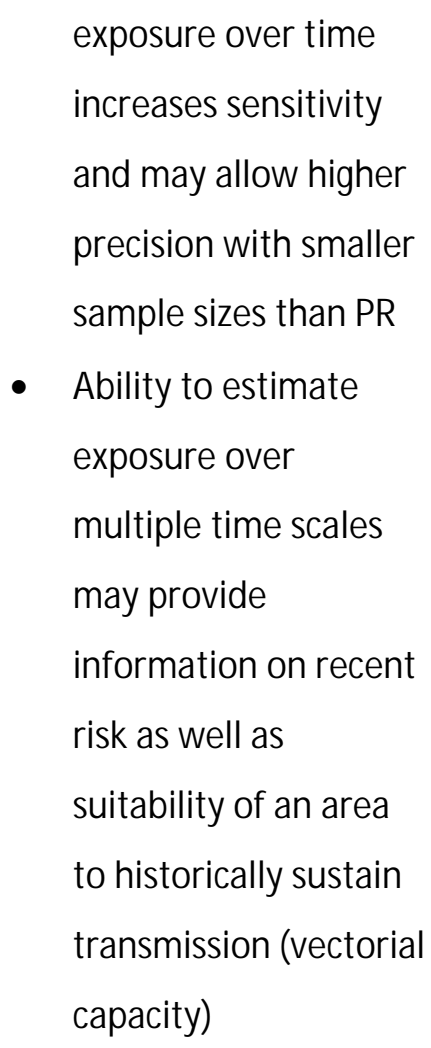

- Ability to estimate

exposure over

multiple time scales

may provide

information on recent

risk as well as

suitability of an area

to historically sustain

transmission (vectorial

capacity)

- Incremental cost to

augment active

surveillance data is

very low

Clinical incidence

- Cheap - data routinely collected at health

facility level

- Provides data over continuous time

- Quality of data highly High (if data are dependent on quality of of sufficient diagnoses, quality)

completeness of reporting and variations in treatment seeking behavior

- Could lead to spurious results without information on whether cases are local or 
imported

- Location data often

restricted to health

facility limiting spatial

resolution of risk maps

- Only useful if spatial

distribution of

symptomatic cases is

reflective of overall

transmission, which may

be driven by

asymptomatic reservoirs

317

318 
320 Figure 1. Serological hotspot and malaria cases in Swaziland 2010-2013. Locations of passively detected

321 local cases during the malaria season 2011-2013 and primary serological 'hotspot' detected in Swaziland

322 from malaria indicator survey (M IS) data collected in 2010 (red ellipse). Each point represents a single

323 case. Figure adapted from Hsiang et al. [19] and Sturrock et al. [45].

324

325 Figure 2. A simulated example illustrating the implications of using varying numbers of pseudo-

326 controls for risk mapping case data. Each figure represents the same geographical area. A - a simulated

327 population surface, B - simulated malaria risk (probability of there being a case), C - simulated case

328 locations; D - a risk map (predicted probability of there being a case) generated using a random forest

329 model with two covariates (simulated elevation and vegetation index - not shown) with 1000 pseudo-

330 controls; E - as per D with 5000 pseudo-controls; F - as per D with 10000 pseudo-controls. Note the

331 difference in level of risk between the simulated malaria risk (B) and its predictions using increasing

332 numbers of pseudo-controls (D-F).

333

334

335 


\section{References}

337

338

339

340

341

342

343

344

345

346

347

348

349

350

351

352

353

354

355

356

357

358

359

360

361

362

363

364

365

366

367

368

369

370

371

372

373

374

375

376

377
1 Gething, P., et al. (2011) A new world malaria map: Plasmodium falciparum endemicity in 2010. Malaria Journal 10, 378

2 Hay, S.l., et al. (2011) Estimating the global clinical burden of Plasmodium falciparum malaria in 2007. PLoS M edicine 7, e1000290

3 Kleinschmidt, I., et al. (2000) A spatial statistical approach to malaria mapping. Int J Epidemiol 29, 355 361

4 Bennett, A., et al. (2013) Mapping malaria transmission intensity in M alawi, 2000-2010. Am J Trop M ed Hyg 89, 840-849

5 Bhatt, S., et al. (2015) The effect of malaria control on Plasmodium falciparum in Africa between 2000 and 2015. Nature 526, 207-211

6 Sturrock, H.J.W., et al. (2013) Reactive case detection for malaria elimination: real-life experience from an ongoing program in Swaziland. PLoS One 8, e63830

7 Stresman, G., et al. (2010) A method of active case detection to target reservoirs of asymptomatic malaria and gametocyte carriers in a rural area in Southern Province, Zambia. M alaria Journal 9, 265 8 Ernst, K.C., et al. (2006) M alaria hotspot areas in a highland Kenya site are consistent in epidemic and non-epidemic years and are associated with ecological factors. M alar J 5, 78

9 Bejon, P., et al. (2014) A micro-epidemiological analysis of febrile malaria in Coastal Kenya showing hotspots within hotspots. Elife 3, e02130

10 Newby, G., et al. (2016) The path to eradication: a progress report on the malaria-eliminating countries. Lancet 387, 1775-1784

11 World Health Organization, G. (2015) World M alaria Report 2015.

12 Tusting, L.S., et al. (2014) M easuring changes in Plasmodium falciparum transmission: precision, accuracy and costs of metrics. Adv Parasitol 84, 151-208

13 Oesterholt, M., et al. (2006) Spatial and temporal variation in malaria transmission in a low endemicity area in northern Tanzania. Malar J 5, 98

14 M bogo, C.M., et al. (2003) Spatial and temporal heterogeneity of Anopheles mosquitoes and

Plasmodium falciparum transmission along the Kenyan coast. Am. J. Trop. M ed. Hyg. 68, 734-742

15 Noor, A.M ., et al. (2014) The changing risk of Plasmodium falciparum malaria infection in Africa: 2000-10: a spatial and temporal analysis of transmission intensity. Lancet 383, 1739-1747

16 Smith, D.L., et al. (2005) The entomological inoculation rate and Plasmodium falciparum infection in African children. Nature 438, 492-495

17 Hay, S., et al. (2008) Measuring malaria endemicity from intense to interrupted transmission. Lancet Infect Dis 8, 369 - 378

18 Jovani, R. and Tella, J. (2006) Parasite prevalence and sample size: misconceptions and solutions.

Trends Parasitol 22, 214 - 218

19 Hsiang, M., et al. (2012) Surveillance for malaria elimination in Swaziland: a national cross-sectional study using pooled PCR and serology. PLoS One 7, e29550

20 Yekutiel, P. (1960) Problems of epidemiology in malaria eradication. Bull. World Health Organ. 22, 669-683

21 Corran, P., et al. (2007) Serology: a robust indicator of malaria transmission intensity? Trends in

Parasitology 23, 575-582 
22 Drakeley, C.J., et al. (2005) Estimating medium- and long-term trends in malaria transmission by using serological markers of malaria exposure. Proceedings of the National Academy of Sciences 102, 51085113 23 Pothin, E., et al. (2016) Estimating malaria transmission intensity from Plasmodium falciparum serological data using antibody density models. Malaria journal 15, 1

24 Yman, V., et al. (2016) Antibody acquisition models: A new tool for serological surveillance of malaria transmission intensity. Scientific reports 6, 19472

25 Bejon, P., et al. (2010) Stable and unstable malaria hotspots in longitudinal cohort studies in Kenya. PLoS M ed 7, e1000304

26 M osha, J., et al. (2014) Hot spot or not: a comparison of spatial statistical methods to predict prospective malaria infections. Malaria Journal 13, 53

27 Ashton, R.A., et al. (2015) Geostatistical modeling of malaria endemicity using serological indicators of exposure collected through school surveys. Am J Trop M ed Hyg 93, 168-177

28 Helb, D.A., et al. (2015) Novel serologic biomarkers provide accurate estimates of recent Plasmodium falciparum exposure for individuals and communities. Proceedings of the National Academy of Sciences 112 , E4438-E4447

29 Ondigo, B.N., et al. (2014) Estimation of recent and long-term malaria transmission in a population by antibody testing to multiple Plasmodium falciparum antigens. J. Infect. Dis. 210, 1123-1132 30 Cook, J., et al. (2011) Serological markers suggest heterogeneity of effectiveness of malaria control interventions on Bioko Island, equatorial Guinea. PLoS One 6, e25137

31 Cook, J., et al. (2012) Sero-epidemiological evaluation of changes in Plasmodium falciparum and Plasmodium vivax transmission patterns over the rainy season in Cambodia. Malaria Journal 11, 86 32 Patil, A., et al. (2009) Defining the relationship between Plasmodium falciparum parasite rate and clinical disease: statistical models for disease burden estimation. M alaria Journal 8, 186

33 Cotter, C., et al. (2013) The changing epidemiology of malaria elimination: new strategies for new challenges. Lancet 382, 900 - 911

34 Sturrock, H.J.W., et al. (2015) Tackling imported malaria: an elimination endgame. The American Journal of Tropical M edicine and Hygiene 93, 139-144

35 Reiner, R.C., et al. (2015) M apping residual transmission for malaria elimination. elife 4:e09520 36 Wesolowski, A., et al. (2012) Quantifying the impact of human mobility on malaria. Science 338, 267 270

37 Tatem, A., et al. (2014) Integrating rapid risk mapping and mobile phone call record data for strategic malaria elimination planning. Malaria Journal 13, 52

38 Greenhouse, B. and Smith, D.L. (2015) M alaria genotyping for epidemiologic surveillance.

Proceedings of the National Academy of Sciences, 201507727

39 Bennett, A., et al. (2014) A methodological framework for the improved use of routine health system data to evaluate national malaria control programs: evidence from Zambia. Population health metrics 12,30 40 Ohrt, C., et al. (2015) Information systems to support surveillance for malaria elimination. The American Journal of Tropical M edicine and Hygiene 41 Zurovac, D., et al. (2012) M obile phone text messaging: tool for malaria control in Africa. PLoS M ed 9, e1001176

42 Gething, P., et al. (2008) Developing geostatistical space-time models to predict outpatient treatment burdens from incomplete national data. Geogr Anal 40, 167 - 188 43 Gething, P., et al. (2007) A local space-time kriging approach applied to a national outpatient malaria dataset. Comput Geosci 33, 1337 - 1350

44 Alegana, V., et al. (2012) Spatial modelling of healthcare utilisation for treatment of fever in Namibia. Int. J. Health. Geogr. 11, 6 
45 Sturrock, H., et al. (2014) Fine-scale malaria risk mapping from routine aggregated case data. Malaria Journal 13, 421

42846 Ceesay, S., et al. (2008) Changes in malaria indices between 1999 and 2007 in The Gambia: a

429 retrospective analysis. Lancet $372,1545-1554$

$43047 \mathrm{Bi}$, Y., et al. (2012) Can slide positivity rates predict malaria transmission? Malaria journal 11, 1-8 malaria in Afghanistan 2006-2009. PLoS One 9, e102304

49 Alegana, V.A., et al. (2013) Estimation of malaria incidence in northern Namibia in 2009 using Bayesian conditional-autoregressive spatial-temporal models. Spatial and Spatio-temporal Epidemiology 7, 25-36

50 Keil, P., et al. (2013) Downscaling of species distribution models: a hierarchical approach. Methods in Ecology and Evolution 4, 82-94

51 Diggle, P.J., et al. (2013) Spatial and spatio-temporal log-Gaussian Cox processes: extending the geostatistical paradigm. Statistical Science 28, 542-563

52 Li, Y., et al. (2012) Log Gaussian Cox processes and spatially aggregated disease incidence data. Stat. M ethods Med. Res. 21, 479-507

53 Prasad, A., et al. (2006) Newer classification and regression tree techniques: bagging and random forests for ecological prediction. Ecosystems 9, 181-199

54 Cohen, J., et al. (2013) Rapid case-based mapping of seasonal malaria transmission risk for strategic elimination planning in Swaziland. Malaria Journal 12, 61

55 Wisz, M. and Guisan, A. (2009) Do pseudo-absence selection strategies influence species distribution models and their predictions? An information-theoretic approach based on simulated data. BM C Ecology 9,8

56 Barbet-M assin, M., et al. (2012) Selecting pseudo-absences for species distribution models: how, where and how many? Methods in Ecology and Evolution 3, 327-338

57 Phillips, S.J. and Elith, J. (2013) On estimating probability of presence from use-availability or presence-background data. Ecology 94, 1409-1419

58 King, G. and Zeng, L. (2013) Case-Control Studies, Inference in. In Encyclopedia of Biopharmaceutical Statistics, Second Edition, pp. 1-13, Taylor \& Francis 59 Pullan, R.L., et al. (2012) Spatial parasite ecology and epidemiology: a review of methods and applications. Parasitology 139, 1870 - 1887

60 Renner, I.W., et al. (2015) Point process models for presence-only analysis. M ethods in Ecology and Evolution 6, 366-379

$61 \mathrm{Ahn}$, J., et al. (2014) A space-time point process model for analyzing and predicting case patterns of diarrheal disease in northwestern Ecuador. Spat Spatiotemporal Epidemiol 9, 23-35 62 Cohen, J., et al. (2010) How absolute is zero? An evaluation of historical and current definitions of malaria elimination. Malar J 9, 213

63 WHO (2007) Malaria Elimination: a field manual for low and moderate endemic countries. World Health Organization

64 M oonen, B., et al. (2010) Operational strategies to achieve and maintain malaria elimination. Lancet $376,1592-1603$

65 WHO (2012) Disease surveillance for malaria elimination: an operational manual. WHO 66 Noor, A., et al. (2013) The receptive versus current risks of Plasmodium falciparum transmission in Northern Namibia: implications for elimination. BM C Infect Dis 13, 184

67 Romi, R., et al. (2012) Assessment of the risk of malaria re-introduction in the Maremma plain (Central Italy) using a multi-factorial approach. M alar J 11, 98 68 Dlamini, S.N., et al. (2015) Assessing the relationship between environmental factors and malaria vector breeding sites in Swaziland using multi-scale remotely sensed data. Geospatial health 10, 88-98 
47469 M achault, V., et al. (2012) Risk mapping of Anopheles gambiae sl densities using remotely-sensed

475 environmental and meteorological data in an urban area: Dakar, Senegal. PLoS One 7, e50674

47670 Gelfand, A.E., et al. (2012) On the effect of preferential sampling in spatial prediction. Environmetrics

$477 \quad 23,565-578$

47871 Diggle, P.J., et al. (2010) Geostatistical inference under preferential sampling. Journal of the Royal

479 Statistical Society: Series C (Applied Statistics) 59, 191-232

48072 Pati, D., et al. (2011) Bayesian geostatistical modelling with informative sampling locations.

481 Biometrika 98, 35-48

48273 Le M enach, A., et al. (2011) Travel risk, malaria importation and malaria transmission in Zanzibar. Sci

483 Rep 1,93

48474 Kelly, G., et al. (2013) A high-resolution geospatial surveillance-response system for malaria

485 elimination in Solomon Islands and Vanuatu. Malar J 12, 108

48675 Larsen, D.A., et al. (2015) M alaria surveillance in low-transmission areas of Zambia using reactive case

487 detection. Malaria journal 14, 1-9

48876 Patel, J.C., et al. (2014) Genetic evidence of importation of drug-resistant Plasmodium falciparum to

489 Guatemala from the Democratic Republic of the Congo. Emerg Infect Dis 20, 932-940

49077 Obaldia, N., et al. (2015) Clonal outbreak of Plasmodium falciparum infection in eastern Panama. J.

491 Infect. Dis. 211, 1087-1096

49278 Preston, M.D., et al. (2014) A barcode of organellar genome polymorphisms identifies the geographic

493 origin of Plasmodium falciparum strains. Nature communications 5, 4052

49479 Yukich, J.O., et al. (2014) A description of malaria sentinel surveillance: a case study in Oromia

495 Regional State, Ethiopia. Malaria journal 13, 1-13

49680 M elles, S.J., et al. (2011) Optimizing the spatial pattern of networks for monitoring radioactive

497 releases. Computers and Geosciences 37, 280-288

49881 Sturrock, H.J.W., et al. (2013) The use of bivariate spatial modeling of questionnaire and parasitology

499 data to predict the distribution of Schistosoma haematobium in coastal Kenya. PLoS Negl. Trop. Dis. 7,

500 e2016

50182 Crainiceanu, C.M ., et al. (2008) Bivariate binomial spatial modeling of Loa loa prevalence in tropical

502 Africa. J Am Stat Assoc 103, 21-37

503

504

505 


\section{Outstanding questions}

506 What combination of metrics is optimal for estimating and predicting malaria transmission in low

507 transmission settings?

508 Which serological markers and metrics are most useful for understanding malaria transmission patterns

509 over short- and medium-term?

510 What are the optimal modeling approaches when dealing with aggregate and individual clinical case

511 data?

512 How can passive surveillance systems accurately and routinely collect information on the locations of

513 clinical cases?

514 What are the best methods to estimate the contribution of imported malaria to local risk?

515 How should malaria risk be predicted in settings where elimination has occurred and which data need to 516 be collected?

517

518 


\section{Trends}

519 Generating malaria risk maps in low transmission settings presents unique challenges which have been 520 inadequately addressed.

521 Improvements in surveillance data and novel application of statistical methods have led to new

522 opportunities for understanding and predicting low transmission risk.

523 Established and new serological measures of exposure offer promise to further augment the accuracy 524 and precision of risk mapping in a cost-effective manner.

525

526 


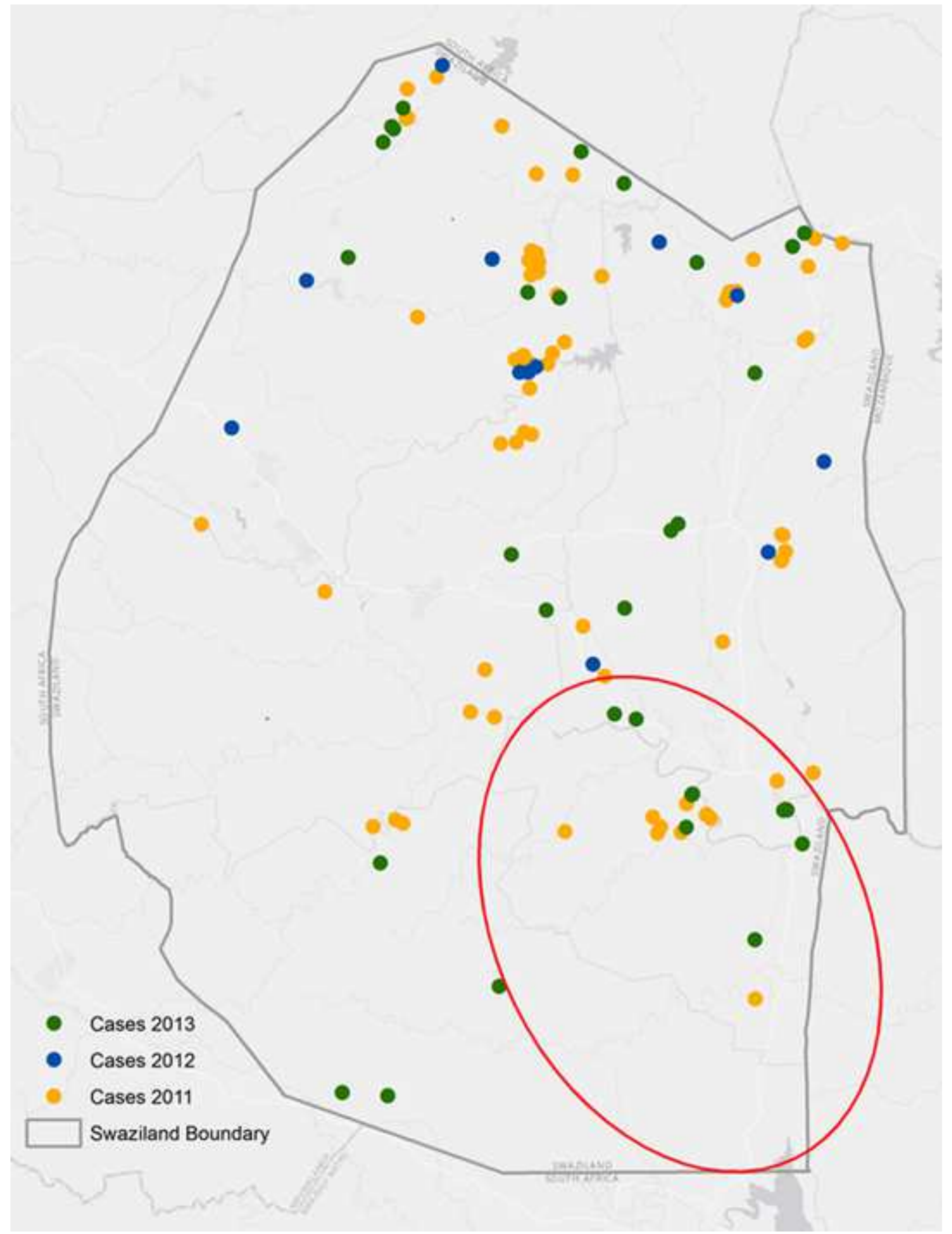

Page 23 of 24 

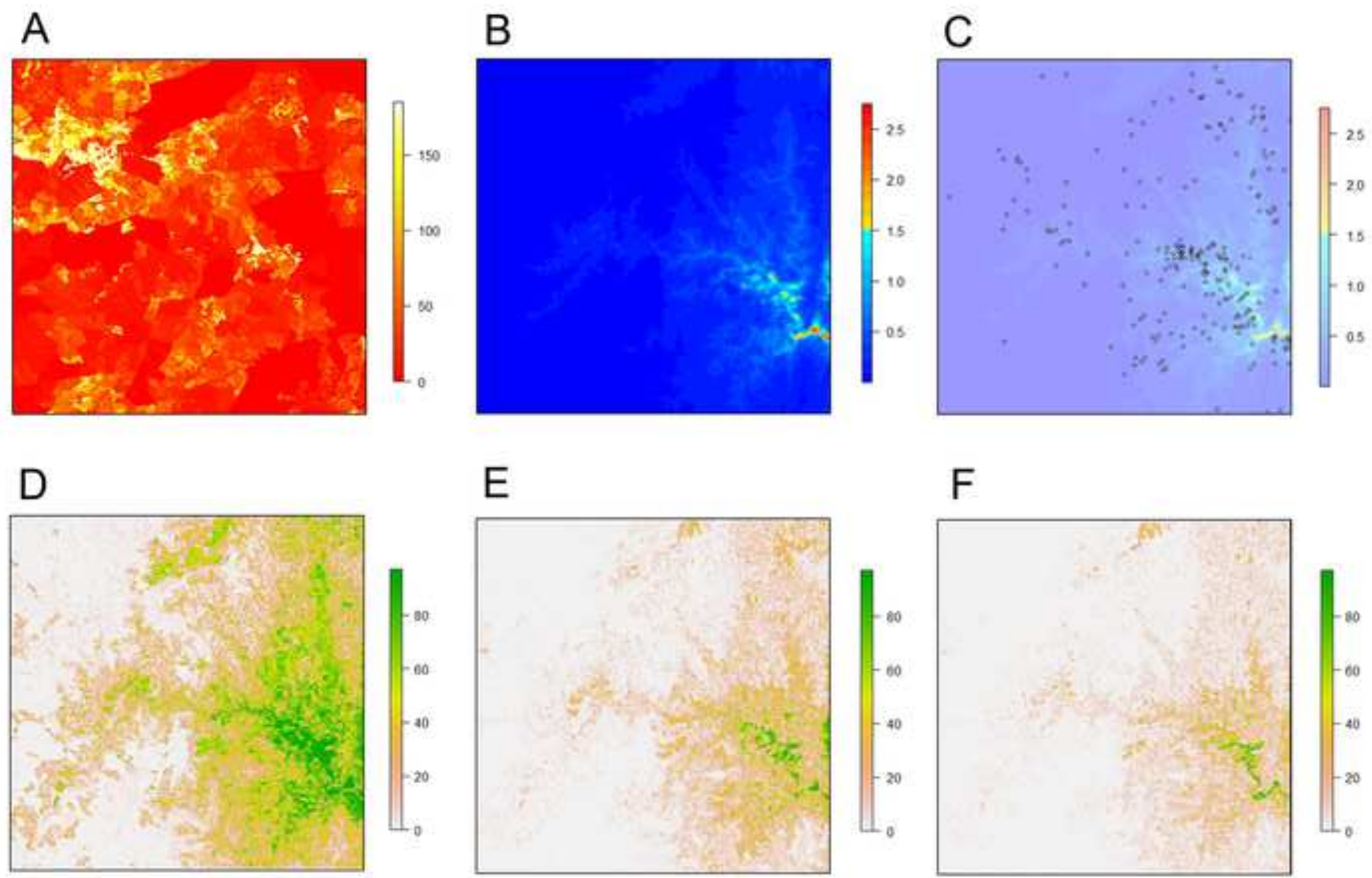